\title{
BMJ Open Parenting acceptance and commitment therapy: a randomised controlled trial of an innovative online course for families of children with cerebral palsy
}

\author{
Koa Whittingham, ${ }^{1,2}$ Jeanie Sheffield, ${ }^{2}$ Roslyn N Boyd ${ }^{1}$
}

To cite: Whittingham K, Sheffield J, Boyd RN. Parenting acceptance and commitment therapy: a randomised controlled trial of an innovative online course for families of children with cerebral palsy. BMJ Open 2016;6:e12807.

doi:10.1136/bmjopen-2016012807

- Prepublication history for this paper is available online. To view these files please visit the journal online (http://dx.doi.org/10.1136/ bmjopen-2016-012807).

Received 25 May 2016 Revised 23 August 2016 Accepted 12 September 2016

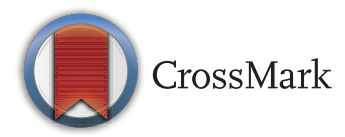

${ }^{1}$ Queensland Cerebral Palsy and Rehabilitation Research Centre, School of Medicine, The University of Queensland, Brisbane, Queensland,

Australia

${ }^{2}$ School of Psychology, The University of Queensland, Brisbane, Queensland, Australia

Correspondence to Dr Koa Whittingham; koawhittingham@uq.edu.au

\section{ABSTRACT}

Introduction: Cerebral palsy (CP) impacts on the entire family in a manner that is long-term, complex and multifactorial. In addition, the quality of the parent-child relationship impacts on many and varied child outcomes, making the provision of easily accessible and evidence-based support to parents of children with $\mathrm{CP}$ a priority. This paper reports the protocol of a randomised controlled trial of an innovative and translatable online intervention, parenting acceptance and commitment therapy (PACT), for families of children with CP. We predict that participating in the PACT programme will be associated with improvements in the parent-child relationship, in child functioning and in adjustment and quality of life for both parent and child.

Methods and analysis: We aim to recruit 66 parents of children (2-10 years old) diagnosed with CP to this study. Families will be randomly assigned to two groups: wait-list control and PACT. PACT is a parenting intervention grounded in acceptance and commitment therapy (ACT) and developed into an online course 'PARENT101 Parenting with Purpose' using the edX platform. All participants will be offered PACT before completion of the study. Assessments will take place at baseline, following completion of PACT and at 6-month follow-up (retention) and will focus on the parent-child relationship, parent and child adjustment and parent and child quality of life. Analysis will follow standard methods for randomised controlled trials using general linear models, specifically analysis of variance or analysis of covariance.

Ethics and dissemination: Ethics approvals have been obtained through the Children's Health Queensland Hospital and Health Service Human Research Ethics Committee (HREC/15/QRCH/115) and The University of Queensland (2015001743). If efficacy is demonstrated, then the PARENT101 course has the potential to be disseminated widely in an accessible manner and at minimal cost. Further, the PACT framework may provide a blueprint for similar online courses with parents in a full range of contexts. Trial registration number: ACTRN12616000351415; Pre-results.

\section{Strengths and limitations of this study}

- This is a randomised controlled trial using Consolidated Standards of Reporting Trials methodology.

- Assessment includes a parent-child observation coded by blinded coders.

- The use of online technology allows for greater flexibility in access and delivery of PARENT101.

- The PARENT101 course has the potential to be disseminated widely in an accessible manner at minimal cost.

- The control condition is a wait-list control and will have access to PARENT101 after the completion of postcourse assessments for ethical reasons.

\section{INTRODUCTION}

\section{Cerebral palsy (CP) is not just a physical} disability

Motor impairments are the clinical hallmark of CP; however, other impairments are common. ${ }^{1}$ Further, the optimal development of children with CP-psychological, social, emotional and cognitive development-is at risk. One in four children with $\mathrm{CP}$ have a behavioural disorder ${ }^{1}$ compared to 1 in 10 typically developing children. ${ }^{2}$ Seven in 10 preschool children with CP show significant delay in social milestones relative to community norms with continued social difficulties at school age. ${ }^{3-5}$ One in five preschool children with CP show a significant delay in selfcare skills. ${ }^{5}$ In the long term, focusing on adults with $\mathrm{CP}$ without intellectual impairment, one in five have not completed high school, 4 in 10 are not living independently, one in two are not competitively employed and two in three are not in a long-term relationship. ${ }^{6}$ Families want support and solutions; however, they receive little support in 
optimising their children's psychological, social, emotional and cognitive development or in optimising child quality of life. Increases in parental responsiveness are a key factor in predicting child developmental benefits of early intervention, ${ }^{7}$ suggesting that parents themselves are pivotal to optimising child development.

\section{Impact of CP on the whole family}

$\mathrm{CP}$ impacts on the entire family in a manner that is longterm, complex and multifactorial. The birth of a child with CP may precipitate complex care responsibilities, financial hardship, limits to occupational attainments, relationship distress, grief and social isolation, all of which have further flow-on effects for the parents, child and family. ${ }^{8}$ In particular, mothers of children with CP experience greater parental stress than mothers of typically developing children. ${ }^{9}$ They are four times more likely to have elevated anxiety symptoms and five times more likely to have moderate depressive symptoms. ${ }^{10}{ }^{11}$ In a cross-sectional study of 818 families of children (812 years) with CP living across Europe, parents were five times more likely to be experiencing very high stress levels than the general population. Interestingly, the magnitude of parenting stress was not associated with severity of motor impairment of the child; ${ }^{12}$ rather, parenting stress was associated with child behavioural and emotional problems. ${ }^{13-16}$ Parents commonly experience an ongoing grieving process, with grief intensifying during transitions and major child and family life events. ${ }^{17}$ Parents of children with CP parent in an emotional context that may include significant stress, ongoing grief, a loss of typical parenting rewards (eg, seeing your child walk for the first time), anxiety about the future and a lack of understanding from the broader community.

The developmental abilities of children impact on the quality of parental caregiving that the child is able to elicit within day-to-day interactions. ${ }^{18}$ Children with neurodevelopmental disabilities, who most benefit from optimal parental support, are unable to effectively elicit optimal parental support. Parents of children with CP need to be proactive, skilled and conscious in their moment by moment parenting choices to provide their children with optimal developmental support. Parenting a child with CP, and optimising their development, involves forward thinking, scaffolding abilities, a commitment to supervision, patience, compassion, behavioural management skills and effort above and beyond the optimal parenting of typically developing children. Further, all of that requires a strong and loving emotional bond and parental psychological resilience.

\section{Parental responsiveness fuels development}

The quality of the parent-child relationship is important for all children, regardless of individual characteristics and impacts greatly on diverse child outcomes, including developmental, emotional, cognitive, behavioural, relational, educational and quality of life outcomes. ${ }^{19-22} \mathrm{~A}$ meta-analysis demonstrated that parent-child relationships with high parental responsiveness-prompt, child-directed, contingent and appropriate parenting-are associated with improved cognitive, emotional, behavioural and social outcomes. ${ }^{23}$ Children who have experienced a warm and emotionally available parent-child bond are more likely to be socially competent and emotionally well-adjusted, establishing stable relationships in later life. ${ }^{24}$ Crucially, language, cognitive reasoning and preacademic skills are learnt through the day-to-day interactions of the parent-child relationship. In a longitudinal observational study with 42 families, the quality of everyday parent-child interactions in the first 3 years of life was found to predict $61 \%$ of the variance in rates of vocabulary growth and use, as well as 59\% of the variance in a child's Standford Binet IQ score at age three. ${ }^{25}$ During everyday relationship-driven encounters, parents provide hour after hour of environmental enrichment: rich, quality experience in verbal skills, $\operatorname{cog}$ nitive reasoning and playful exploration. Parental responsiveness can be conceptualised as nature's dosecontrol system for environmental enrichment, ${ }^{26}$ enabling the child to obtain 'just right' stimulation for learning and development organically in day-to-day life via parent-child interaction and the flow-on effects of such interaction. Nowhere is this more important than in the parent-child relationships of children with developmental delays or disabilities. These relationships may be built and enhanced through the targeted assistance of various psychological approaches.

\section{Acceptance and Commitment Therapy (ACT): a promising approach}

ACT, said as the word 'act' is now an accepted part of the broad cognitive-behavioural therapy (CBT) approach. ACT incorporates: mindfulness or deliberate non-judgemental attention to moment to moment experience, experiential acceptance or ongoing nonjudgemental contact with psychological events such as difficult emotions, cognitions and memories and vital living or engagement in meaningful, values-driven activities. $^{27}$ The goal of ACT is to increase psychological flexibility: the ability to persist or change one's behaviour, with full awareness of the situational context and one's own present-moment experience, in the service of chosen values. ACT and related psychological therapies have a growing evidence base for a diverse range of issues, for example, occupational stress, depression, anxiety and smoking cessation. ${ }^{28-31}$ Meta-analyses, examining the efficacy of ACT and related psychological therapies with varying dosages $\left(30.0 \pm 29.8\right.$ hours in $\left.^{30}\right)$, and targeting a variety of issues, have identified moderate effect sizes ranging from 0.5 to $0.75 .^{28-31}$ Psychological flexibility, the clinical target of ACT, predicts levels of parental psychological symptoms, experienced parenting burden and symptoms of grief in parents of children with $\mathrm{CP}^{17}$ as well as parental psychological symptoms, maternal bonding and responsiveness in mothers of infants born preterm. ${ }^{32}$ 
Face-to-face parenting intervention incorporating ACT is beneficial

A parenting intervention incorporating ACT was shown to be efficacious within a three-group $\mathrm{RCT}^{33-35}$ Sixty-seven parents of children (mean age 5.3) with CP participated in the trial. Parents were randomly assigned to: wait-list control, the parenting intervention Stepping Stones Triple P or to a combined Stepping Stones Triple $\mathrm{P}$ and ACT group. Families receiving Stepping Stones Triple $\mathrm{P}$ alone, compared to wait-list, showed improvements in child behaviour and emotional symptoms. Families receiving the combined Stepping Stones Triple $\mathrm{P}$ and ACT intervention, compared to wait-list control, showed improvements in child behaviour, child hyperactivity, child functional mobility performance, child quality of life, parenting style and parental adjustment. During the randomised controlled trial (RCT), face-to-face delivery was identified as a barrier to effective translation requiring an innovative solution to capitalise on the potential benefits from the ACT-based approach.

\section{Online therapy is effective and translatable}

A meta-analysis of web-based psychological therapy indicates that web-based delivery is effective with an average effect size across intervention types and measures of 0.53 consistent with a medium effect and with greater effect sizes of 0.83 for cognitive-behavioural interventions. ${ }^{36}$ Further, 14 RCTs were identified that directly compared web-based psychological therapy with traditional face-to-face psychological therapy and no significant differences between the two treatment modalities could be found. Online delivery modalities may be an ideal vehicle for low-cost and sustainable knowledge translation directly to parents in all situations, but specifically those families with children with disabilities who may require extra time and assistance, therefore reducing the capacity to access traditional forms of support.

If found effective, parenting acceptance and commitment therapy (PACT) could be easily and rapidly disseminated population-wide. Potentially, families of children with $\mathrm{CP}$ in different countries could access immediate evidence-based psychological support, from first receiving diagnosis onwards, to promoting parent and child flourishing and to harnessing existing strengths within the parent-child relationship to foster long-term developmental gains for the child. PACT is flexible and family-centred, leveraging the understanding, skills and the day-to-day interactions that already exist within the family system for the benefit of both child and family and placing the parents as the central experts in their own child's behaviour.

\section{Aim}

Our aim is to demonstrate the efficacy of an innovative, translatable-by-design online parenting support package: PACT, said as the word 'PACT' for families of young children (2-10 years of age) with CP. PACT will be delivered in the form of an online course for parents: PARENT101: Parenting with Purpose.

\section{Hypotheses}

We predict that participating in the PACT course, PARENT101: Parenting with Purpose, will be associated with improvements in the parent-child relationship and in parent and child adjustment and quality of life. Following baseline assessment, outcomes will be assessed postintervention after the final review session and at 6 months postintervention (retention of treatment effects).

\section{METHODS AND ANALYSIS \\ Design}

The study is a RCT following Consolidated Standards of Reporting Trials (CONSORT) guidelines. Parents of children with $\mathrm{CP}$ will be randomly allocated to one of two groups:

1. Online PACT in the form of the online course: PARENT101: Parenting with Purpose

\section{Wait-list control}

Follow-up will occur at 6 months postintervention (retention). The wait-list condition will be offered the intervention after completion of the review assessment.

\section{Recruitment}

We aim to recruit 66 parents of children (2-10 years old) diagnosed with CP. Families of children with CP will be recruited through the Queensland Cerebral Palsy and Rehabilitation Research Centre, hospitals around Australia, the Australian Cerebral Palsy Register and word of mouth. Since PACT uses online delivery, there are no impediments to participants being recruited from across Australia including in regional areas, making this project highly feasible.

Exclusion criteria: Since PACT uses integrated webbased delivery, parents are required to have: (1) reliable internet access at home (eg, ADSL) and be committed to maintaining internet access for the duration of the study; (2) a mobile phone for receiving text messages that they are asked to check regularly throughout the study; (3) an email address for receiving emails that they are asked to check several times a week throughout the study; and (4) access to Skype.

Sample size: To have sufficient power to detect an effect size of 0.40 (power 0.8 ), a total sample size of 52 is required. If a conservative retention rate of $80 \%$ is assumed, this leads to a total of 66 families to be recruited.

\section{The PACT intervention}

PACT includes ACT techniques already established in an RCT as effective with families of children with CP, including mindfulness, experiential acceptance and cognitive defusion. ${ }^{33-35}$ It includes some techniques from the established parenting literature but focuses on strategies 
to enhance the parent-child relationship and promote parental emotional responsiveness. An online course was created using the edX platform, an open source course management system created by founding partners Harvard and MIT (http://www.edx.org/). The edX platform is used to host Massive Open Online Courses and allows for the online delivery of a variety of educational courses that can be accessed from anywhere that has internet availability.

Within the edX framework, the PACT programme has been developed to give it the look and accessibility of a course and has been named PARENT101: Parenting with Purpose. PARENT101 incorporates: virtual written text, video presentations, screen-based interactive activities, journal activities, reflections, guided experiential and mindfulness exercises and participation in a moderated discussion board. In addition, research participants will be offered Skype sessions with a therapist as well as reminder text messages and emails. PARENT101 is presented as three modules plus a review session 4 weeks after the final module. Modules are scheduled to be completed each fortnight, with a 4-week break after that to allow for practice of the materials from the course. Overall, the course lasts for 10 weeks (6 weeks for modules plus review 4 weeks later) in total, with parents able to move through each module's content with some flexibility. Access to PARENT101 will be restricted to study participants until efficacy is established.

\section{Study procedure}

After obtaining ethical approval, a recruitment strategy was developed to maximise opportunity for interested parents to participate. This involves recruiting through a variety of public hospitals, CP-related organisations and potentially social media sites relevant to parents of children with CP. In hospitals, relevant clinicians have agreed to develop recruitment protocols that introduce the study details and provide mechanisms to register interest in the study. In other settings, flyers/promotional letters will be disseminated with contact details of the researchers for interested participants.

Following registration of interest by individuals, they will contacted by researchers to assess eligibility for participation. After provision of written informed consent, parents will be asked to complete all baseline measures, including recording a parent-child interaction for observation, before being randomly allocated to either intervention or control. Randomisation will be applied using a computer-generated block randomisation. The initial randomisation sequence will be managed by an individual outside of the study. Following recruitment, the research manager will determine each participant's status by opening an opaque envelope. Although the researchers will not be involved in the initial creation of the randomisation sequence, owing to the study design they will not be blind to participants' condition status. Families allocated to the PACT condition will then receive access to the PARENT101 course for immediate enrolment and the control condition participants will be advised of their timelines. In the interests of equity and retaining participants, the control condition participants will be offered access to the course after completion of the postreview assessment.

Assessment will be conducted at baseline, postreview (10 weeks after course initiation) and at 6 months postintervention follow-up. All written assessment measures will be completed by parents. Parents will also have the opportunity to provide course evaluation feedback after each module and at the end of the review session.

\section{Measures}

A Family Background Questionnaire will be developed, tailoring existing standardised measures to assess general demographic variables such as SES and parental education and family factors specific to the CP context.

Gross Motor Function Classification System is a parent rating measure using a five-level system to classify children into their age-specific gross motor ability. ${ }^{37}$ It is valid and reliable and frequently used to classify functional abilities of children with physical disability.

Emotional Availability Scales (EAS; Z Biringen, J Robinson, $R$ Emde. Emotional Availability (EA) Scales. 3rd edn. Unpublished manual, $1998^{38}$ ) The EAS is the primary outcome measure. Parents will be asked to video record a 20 min naturalistic observation of the parent-child relationship in the home. Families will be able to send their recordings through a secure File Transfer Protocol) connection. It will be scored by an independent rater, blind to the intervention condition, using the EAS. The independent rater will be trained in the EAS. The EAS is a dyadic measure, that is, it measures the quality of the relationship itself across six scales: parental sensitivity, parental structuring, parental nonintrusiveness, parental non-hostility, child responsiveness and child involvement. The EAS also generates a global relationship quality rating. The scale has high inter-rater reliability for parental responsiveness $(0.96)$, involving (0.87), sensitivity (0.93) and structuring (0.76)

Interpersonal Mindfulness in Parenting $\left(I M P^{39}\right)$ scale is a 10-item measure of a parent's ability to maintain present-centred attention and emotional awareness during parent-child interactions. Parents respond on a five-point Likert scale to a series of statements. The Intramuscular-P scale produces four subscales: presentcentred attention in parenting, present-centred emotional awareness in parenting, non-reactivity/lowreactivity in parenting and non-judgemental acceptance in parenting. The IMP scale has adequate concurrent and discriminant validity. ${ }^{40}$

Emotional Availability-Self Report $\left(E A-S R^{41}\right)$ is a 32-item parent-report measure of emotional availability within the parent-child relationship. It has excellent reliability and validity. The EA-SR produces five subscales: mutual attunement, affect quality, capacity to involve the parent, intrusiveness and hostility. 
Acceptance and Action Questionnaire $\left(A A Q-7^{42}\right)$. This 7-item version of the Acceptance and Action Questionnaire measures experiential avoidance, that is, attempts to control the form, frequency or situational sensitivity of private events such as memories, cognitions and emotions, particularly when doing so causes harm. It produces a single total scale and can be scored either so that high scores reflect high experiential acceptance or so that high scores reflect high experiential avoidance. A series of statements is rated on a seven-point scale. The AAQ has satisfactory internal consistency $(\alpha=0.79)$.

Depression Anxiety Stress Scale (DASS-21 ${ }^{43}$ ) is a 21-item questionnaire that assesses symptoms of depression, anxiety and stress in adults. Respondents rate items on a four-point Likert scale reflecting how much the statement applied to them in the past week. The DASS produces three subscales each with good internal consistency: the depression $(\alpha=.91)$, anxiety $(\alpha=.84)$ and stress $(\alpha=.90)$ scales. The DASS also has good discriminant and concurrent validity (Brown, Chorpita, Korotitsch, and Barlow, 1997; Lovibond and Lovibond, 1995).

Personal Well-being Index: $\left(P W ;^{44}\right)$ is an eight-item measure of personal well-being in adults. Respondents rate their degree of satisfaction with various domains of living on a 10-point Likert scale. The responses are summed to create an average score representing subjective well-being. Respondents can also be asked to rate their life as a whole. The PWI has good validity and internal consistency $\left(\alpha>0.70 ;{ }^{44} 45\right)$.

Strengths and Difficulties Questionnaire $\left(S D Q^{46}\right)$ is a 25-item parent-report measure of child behaviour and adjustment in which the frequency of behaviours is rated on a three-point Likert scale. The SDQ produces five subscales: emotional symptoms, conduct problems, inattention/hyperactivity, peer problems and prosocial behaviour (range 0-10). It produces a total difficulties score (range 0-40) that has been found to have adequate internal reliability $(\alpha=.76)$ and test-retest reliability ( $\mathrm{r}=.86)$ as well as discriminant and concurrent validity. ${ }^{47}$ The SDQ is widely used in CP research. ${ }^{16}{ }^{48}$

$C P Q O L$-Child ${ }^{49}$ is a 66 -item parent-report measure of child quality of life that is specifically developed for use in children with CP. It measures quality of life across several aspects of the child's life: physical well-being, social well-being, emotional well-being, school, service access and social acceptance. Higher scores on each scale reflect a higher quality of life in that domain. It has good concurrent validity, internal consistency $(\alpha=0.76-0.89)$ and test-retest reliability $(r=0.80-0.90)$.

\section{What do parents think of PACT?}

A key aspect of the study is ongoing parental evaluation of the course and its suitability for their situation. Client satisfaction with PARENT101 content and format will be measured using questions embedded into the course after completion of each module and review. The questions will ask parents to rate various aspects of PARENT101 on 10-point Likert scales as well as give qualitative feedback. This feedback will be examined in the light of unintended adverse effects and appropriate steps will be taken if any are noted.

\section{Data management}

Questionnaire data will be collected online through Qualtrics and uploaded into SPSS. Observational data will be entered manually. All data will be stored securely on the university network.

\section{Statistical analysis}

Analysis will follow standard methods for randomised controlled trials using comparisons between the two groups. Initially, the two groups will be assessed to determine if they differ on key variables at baseline and covariates will be used to control for this in postintervention analyses if necessary. The protocol of intention-to-treat analysis will be followed in order to minimise inflation of treatment effects consistent with CONSORT guidelines. The experimental unit will be the family represented by parent-reported data. Attrition analysis will be conducted, although strategies will be used in order to minimise missing data and attrition. Data will be analysed using SPSS. The hypotheses relating to intervention efficacy will be tested using general linear models, specifically via analysis of variance or analysis of covariance. Where continuous data exhibit substantial skewness not overcome by transformation, non-parametric methods will be used for simple comparisons.

\section{Ethics and dissemination}

This trial has been registered with the Australian New Zealand Clinical Trials Registry (ACTRN12616000351415). Study results will be disseminated through publication in scientific journals and presentation at relevant conferences as well as directly to research participants. If efficacious, PARENT101 has the potential to be widely disseminated.

\section{DISCUSSION}

This study aims to evaluate whether an innovative, flexible, easily accessible and translatable course grounded in ACT and created using the edX platform for online delivery will be efficacious in enhancing the parentchild relationship in families with children with CP. If found to demonstrate efficacy, the PARENT101 course has the potential to be disseminated widely in an accessible manner at minimal cost and may also provide a blueprint for use of similar online courses with parents in a full range of contexts and situations. In addition, this course could also be provided to organisations and services as a means of providing support to those parents who may not be able to access traditional forms of face-to-face assistance. 
Acknowledgements This work was supported by a Cerebral Palsy Alliance Grant (PG0214) and a National Health and Medical Research Council postdoctoral fellowship (K.W. 631712).

Contributors KW took a lead role in designing this study and writing the protocol. Both KW and JS, working together, created PARENT101. JS and RNB both contributed their expertise on study design and contributed to drafts of the protocol.

Funding This work was supported by a Cerebral Palsy Alliance Grant (PG0214), a National Health and Medical Research Council postdoctoral fellowship (K.W. 631712) and a National Health and Medical Research Council fellowship (R.B. 1105038)

Competing interests None declared.

Ethics approval Children's Health Queensland Hospital and Health Service Human Research Ethics Committee (HREC/15/QRCH/115); The University of Queensland Research Ethics Committee (2015001743).

Provenance and peer review Not commissioned; externally peer reviewed.

Open Access This is an Open Access article distributed in accordance with the Creative Commons Attribution Non Commercial (CC BY-NC 4.0) license, which permits others to distribute, remix, adapt, build upon this work noncommercially, and license their derivative works on different terms, provided the original work is properly cited and the use is non-commercial. See: http:// creativecommons.org/licenses/by-nc/4.0/

\section{REFERENCES}

1. Novak I, Hines M, Goldsmith S, et al. Clinical prognostic messages rom a systematic review on cerebral palsy. Pediatrics 2012;130:1285-312.

2. Costello JE, Egger $\mathrm{H}$, Angold $\mathrm{A}$. Ten year research update review: the epidemiology of child and adolescent psychiatric disorders: I. methods and public health burden. J Am Acad Child Adolesc Psychiatry 2005;44:972-86.

3. Parkes J, White-Koning, Mccullough N, et al. Psychological problems in children with hemiplegia: a European multicentre survey. Arch Dis Child 2009:94:429-33.

4. Whittingham K, Fahey M, Rawicki B, et al. The relationship between motor abilities and early social development in a preschool cohort of children with cerebral palsy. Res Dev Disabil 2010;31:1346-51.

5. Gehrmann FE, Coleman A, Weir KA, et al. School readiness of children with cerebral palsy. Dev Med Child Neurol 2014;56:786-93.

6. Frisch D, Msall ME. Health, functioning, and participation of adolescents and adults with cerebral palsy: a review of outcomes research. Dev Disabil Res Rev 2013;18:84-94.

7. Mahoney G, Boyce G, Fewell RR, et al. The relationship of parent-child interaction to the effectiveness of early intervention services for at-risk children and children with disabilities. Topics Early Child Spec Educ 1998;18:5-17.

8. Bourke-Taylor H, Howie L, Law M. Barriers to maternal workforce participation and relationship between paid work and health. J Intellect Disabil Res 2011;55:511-20.

9. Rentinck ICM, Ketelaar M, Jongmans MJ, et al. Parents of children with cerebral palsy: a review of factors related to the process of adaptation. Child Care Health Dev 2007;33:161-9.

10. Barlow JH, Cullen-Powell LA, Cheshire A. Psychological well-being among mothers of children with cerebral palsy. Early Child Dev Care 2006;176:421-8.

11. Lach LM, Kohen DE, Garner RE, et al. The health and psychosocial functioning of caregivers of children with neurodevelopmental disorders. Disabil Rehabil 2009;31:607-18.

12. Parkes J, Caravale BARBAR, Marcelli MARCO, et al. Parenting stress and children with cerebral palsy: a European cross-sectional survey. Dev Med Child Neurol 2011;53:815-21.

13. Ketelaar M, Volman MJM, Gorter JW, et al. Stress in parents of children with cerebral palsy: what sources of stress are we talking about? Child Care Health Dev 2008;34:825-9.

14. Abbeduto L, Seltzer MM, Shattuck $P$, et al. Psychological well-being and coping in mothers of youths with autism, down syndrome or fragile X syndrome. Am J Ment Retard 2004;109:237-54.

15. Plant KM, Sanders MR. Predictors of care-giver stress in families of preschool-aged children with developmental disabilities. J Intellect Disabil Res 2007;51:109-24.
16. Parkes J, Mccullough N, Madden A, et al. The health of children with cerebral palsy and stress in their parents. $J$ Adv Nurs 2009;65:2311-23.

17. Whittingham K, Wee D, Sanders MR, et al. Sorrow, coping and resiliency: parents of children with cerebral palsy share their experiences. Disabil Rehabil 2013;35:1447-52.

18. Howe D. Disabled children, parent-child interaction and attachment Child Fam Soc Work 2006;11:95-106.

19. Gottman JM, Katz LF, Hoover C. Meta-emotion: how families communicate emotionally. Mahwah, NJ: Lawrence Erlbaum Associates, 1997.

20. Patterson GR. Coercive family process. Vol 3. Oregon: Castalia Publishing Company, 1982.

21. Landry SH, Smith KE, Swank PR. Responsive parenting: establishing early foundations for social, communication and independent problem-solving skills. Dev Psychol 2006;42:627-42.

22. Aran A, Shalev RS, Biran G, et al. Parenting style impacts on quality of life in children with cerebral palsy. J Pediatr 2007;151:56-60.

23. Eshel N, Responsive parenting: interventions and outcomes. Bull World Health Organ 2006;84:991-8.

24. Sroufe AL. Attachment and development: a prospective, longitudinal study from birth to adulthood. Attach Hum Dev 2005;7:349-67.

25. Hart B, Risley TR. Meaningful differences in the everyday experience of young Americian children. Baltimore: Paul H Brookes Publishing Co, 1995.

26. Morgan C, Novak I, Badawi N. Enriched environments and motor outcomes in cerebral palsy: systematic review and meta-analysis. Pediatrics 2013;132:e735-46.

27. Hayes SC. Acceptance and commitment therapy and the new behavior therapies. In: Hayes SC, Follette VM, Linehan MM, eds Mindfulness and acceptance expanding the cognitive-behavioral tradition. New York: The Guilford Press, 2004:1-29.

28. Grossman P, Niemann L, Schmidt S, et al. Mindfulness-based stress reduction and health benefits a meta-analysis. J Psychosom Res 2004;57:35-43.

29. Baer RA. Mindfulness training as a clinical intervention: a conceptua and empirical review. Clin Psychol 2003;10:125-43.

30. Ost L-G. Efficacy of the third wave of behavioral therapies: a systematic review and meta-analysis. Behav Res Ther 2008;46:296-321.

31. Ruiz FJ. Acceptance and Commitment Therapy versus Traditional Cognitive Behavioral Therapy: a systematic review and meta-analysis of current empirical evidence. In J Psychol 2012;12:333-57.

32. Evans $\mathrm{T}$, Whittingham $\mathrm{K}$, Boyd R, What helps the mother of a preterm infant become securely attached, responsive and well-adjusted? Infant Behav Dev 2012;35:1-11.

33. Whittingham $\mathrm{K}$, Sanders $\mathrm{M}$, Mckinlay $\mathrm{L}$, et al. Interventions to reduce behavioral problems in children with cerebral palsy: an RCT. Pediatrics 2014:133:e1249-57.

34. Whittingham K, Sanders M, Mckinlay L, et al. Stepping Stones Triple $P$ and Acceptance and Commitment Therapy for parents of children with cerebral palsy: trial protocol. Brain Impairment 2013;14:270-80.

35. Whittingham K, Sanders MR, Mckinlay L, et al. Parenting intervention combined with Acceptance and Commitment Therapy: a trial with families of children with cerebral palsy. J Paediatr Psychol 2016;41:531-42.

36. Barak A, Hen L, Boniel-Nissim M, et al. A comprehensive review and a meta-analysis of the effectiveness of internet-based psychotherapeutic interventions. J Technol Hum Serv 2008;26:109-60.

37. Palisano R, Rosenbaum P, Walter S, et al. Development and reliability of a system to classify gross motor function in children with cerebral palsy. Dev Med Child Neurol 1997;39:214-23.

38. Biringen Z, Fidler DJ, Barrett KC, et al. Applying the Emotional Availability Scales to children with disabilities. Infant Ment Health $J$ 2005;26:369-91.

39. Duncan LG. Assessment of mindful parenting among parents of early adolescents: development and validation of the interpersonal mindfulness in parenting scale in Human Development and Family Studies. Pennsylvania: Pennsylvania State University, 2007.

40. Duncan LG, Coatsworth JD, Greenberg MT. A model of mindful parenting: implications for parent-child relationships and prevention research. Clin Child Fam Psychol Rev 2009;12:255-70.

41. Vliegen N, Luyten $\mathrm{P}$, Biringen Z. A multimethod perspective on emotional availability in the postpartum period. Parent Sci Pract 2009;9:228-43.

42. Bond FW, Bunce D. The role of acceptance and job control in mental health, job satisfaction and work performance. J Appl Psychol 2003;88:1057-67.

43. Lovibond PE, Lovibond SH. The structure of negative emotional states: comparison of the Depression Anxiety Stress Scales (DASS) 
with the Beck Depression and Anxiety Inventories. Behav Res Ther 1995;33:335-43.

44. International Wellbeing Group. Personal Wellbeing Index. 5th edn. Melbourne: Australian Centre on Quality of Life, Deakin University, 2013.

45. Lau ALD, Cummins RA, Mcpherson W. An investigation into the Cross-Cultural Equivalence of the Personal Wellbeing Index. Soc Indic Res 2005;71:403-30.

46. Goodman R. The strengths and difficulties questionnaire: a research note. J Child Psychol Psychiatry 1997;38:581-6.
47. Goodman R, Scott S. Comparing the strengths and difficulties questionnaire and the child behavior checklist: is small beautiful?. $J$ Abnorm Child Psychol 1999;27:17-24.

48. Parkes J, Mccusker C. Common psychological problems in cerebral palsy. J Paediatr Child Health 2008;18: 427-31.

49. Waters E, Davis E, Mackinnon A, et al. Psychometric properties of the quality of life questionnaire for children with Cerebral Palsy. Dev Med Child Neurol 2007;49:49-55. 